\title{
Simply the best: the 2004 ERS/ATS web guidelines for management of COPD
}

Would Sir/Madam prefer Spanish [1], French [2], German [3], Australian [4], Canadian [5] or even Japanese [6]? No this is not a choice of fine wines for the connoisseur, but a selection from the menu of chronic obstructive pulmonary disease (COPD) management guidelines available to the clinician. So, which do you use and why should you choose the new European Respiratory Society (ERS)/American Thoracic Society (ATS) guidelines? The reason is that they are simply the best!

An extravagant claim perhaps but one that is, I believe, justified by the facts. The ERS/ATS Task Force position paper on the diagnosis and treatment of patients with COPD has only just been published [7]. As such they are the most up-to-date guidelines in the world literature, using clinical trial data not always available to existing guidelines. The ERS/ATS publication utilises the expert knowledge of the two biggest and most important respiratory societies in the world. The guideline team is totally independent not only from pharmaceutical company sponsorship but also any national interference or bias.

These concepts are exemplified by the section on the pharmacology therapy of the stable COPD patient, an area of traditional confusion and conflicting advice, but where several new studies have been released in the last year that help clarify matters. Within the guidelines, new evidence is endorsed for the efficacy of the long acting anti-cholinergic tiotropium in reducing exacerbation rates and hospitalisation of COPD patients. In the same manner, inhaled corticosteroids may also help reduce exacerbation rates, but it is also recommended that they be withdrawn if thought to be ineffective. Recent studies demonstrating the efficacy of combining inhaled long-acting $\beta_{2}$ agonists with inhaled steroids are also presented with definite improvements in both symptoms and lung function above those achieved with either drug given alone. The proven beneficial effects on symptoms, quality of life and lung function of the main classes of bronchodilators, both short- and long-acting, together with methylxanthines, is presented simply and clearly in a concise table to provide the clinician with an at-a-glance summary of all the available evidence. To conclude this section, the guidelines provide a four-step flow chart for the symptomatic management of stable COPD that applies the evidence base described above (fig. 1).

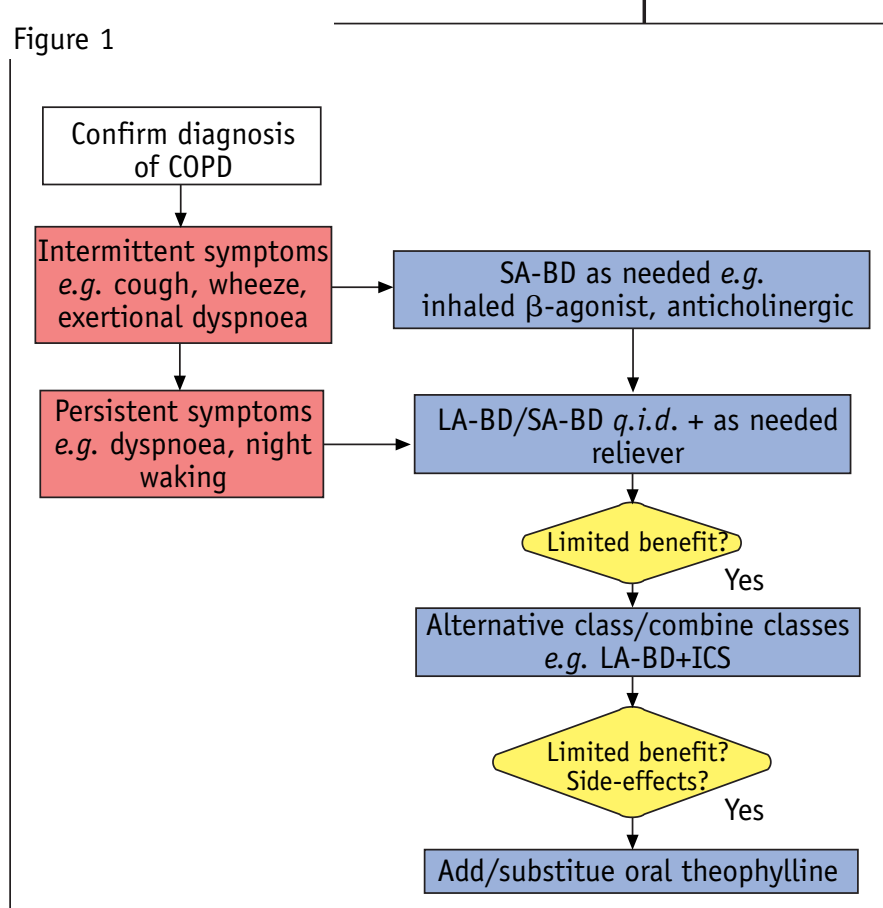

\section{Roberts}

Consultant Physician and Professor of Medical Education Whipps Cross University Hospital with Queen Mary's College Westfield and the University of London Leytonstone

E11 1NR

UK

Fax: 442085356709

E-mail:

michael.roberts@whippsx.nhs.uk
Figure 1

Algorithm for pharmacological treatment of COPD. Reproduced with permission from [7]. 
Figure 2

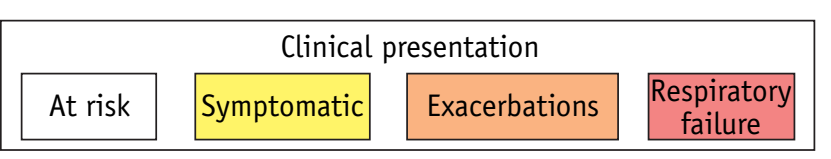

Figure 2

Continuum of care for COPD; the main navigation tool for the COPD guidelines.

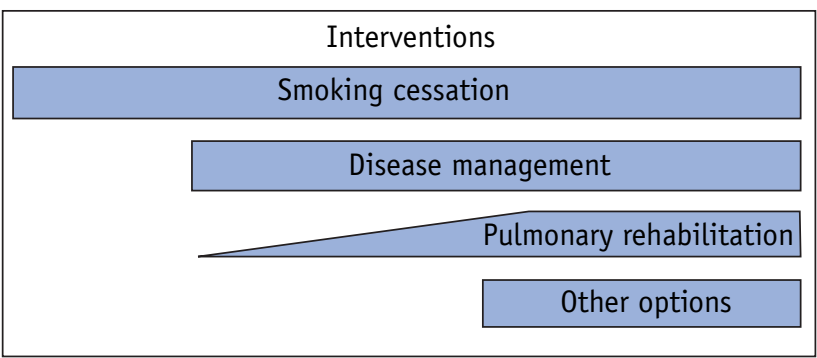

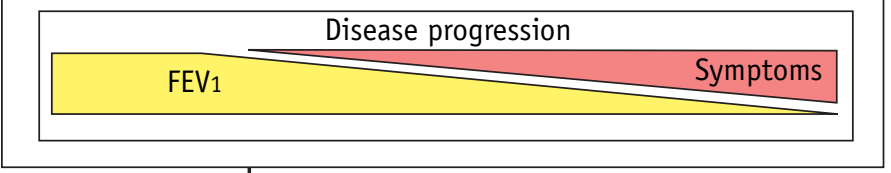

Not only are the guidelines up to date and specifically relevant for the practicing European or American physician but they are also concise yet wide-ranging. The main topics covered are not confined to those of pharmacological management of the stable and acute patients, but a variety of other important but often neglected areas are included. There are stimulating sections on sleep management, consideration of fitness to fly, and integration of secondary and primary care for the COPD patient pathway.

One particular issue that is not shirked is end of life care. The guidelines include a controversial section on the ethical and palliative care issues that should be faced when end-of- life decisions must be made. Specifically, the position statement places the patient at the centre of decision-making concerning mechanical ventilation and strongly suggests that clinicians should be encouraging their stable patients to consider these issues in advance of an acute deterioration. Thought provoking if a little uncomfortable for some clinicians, these highlighted matters require careful consideration in the context of the guidelines and should initiate a debate in all care centres on the guidance issued to clinicians when contemplating assisted ventilation in acute COPD exacerbations. The unacceptable variation in ventilation rates between units and locally derived indications for intubation currently observed must surely come to an end if this lead is followed.

If you need any more persuading then go to the web! These guidelines are unique in being designed for dissemination via the web. Log on to www.ersnet.org/COPD-Guidelines and let the main navigation tool help to guide you to your area of interest (fig. 2).

The new ERS/ATS COPD guidelines for the diagnosis and management of COPD. Upto-date, clearly presented and designed to meet the needs of the clinician of 2004, simply the best! See your local internet service provider for details! e-learning project E-ProLearn linked to these guidelines? EProLearn is a web-based programme that provides a selfassessment exercise designed to help you define the areas in which your knowledge may need updating. Short summaries of relevant guideline sections are electronically provided to the user. A further self-assessment can then be undertaken to help assure the learner that they have taken on board the new information. If you find reading guidelines from cover to cover impossible try this new approach to targeted learning at www.ersnet.org/eprolearn but hurry this is a temporary site only. 